\title{
METHODS OF MATHEMATICAL MODELLING OF THE PROCESSES OF ELECTRODE METAL DROP FORMATION AND TRANSFER IN CONSUMABLE ELECTRODE WELDING (Review)
}

\author{
A.P. SEMYONOV \\ E.O. Paton Electric Welding Institute, NASU \\ 11 Bozhenko Str., 03680, Kiev, Ukraine. E-mail: office@paton.kiev.ua
}

\begin{abstract}
Processes of welding wire heating and melting, electrode metal drop formation and transfer in consumable electrode welding largely determine welding efficiency and quality. In its turn, the nature of metal melting and transfer with this welding process is determined by a large number of such physical phenomena as heat and mass transfer, gas(hydro)dynamics, electromagnetic processes, running in arc plasma, on the surface and in the volume of molten electrode metal-drop. This paper gives a review of currently available methods of theoretical investigation and mathematical modelling of the above processes, allowing prediction of such characteristics of electrode metal transfer as drop volume and shape, their thermal and gas-dynamic state, detachment frequency, etc. Advantages and disadvantages of the considered models are analyzed and main directions of their further development are outlined. 37 Ref., 11 Figures.
\end{abstract}

Keywords: consumable electrode welding, mathematical modelling, electrode metal drop formation

Interest to the problem of metal transfer in consumable electrode welding is due to a number of causes. It is known that formation of electrode metal drop can be accompanied by its overheating, leading to considerable loss of alloying elements contained in welding wire, bulk boiling and spattering of drop metal, closing of arc gap, etc. In addition, metal transfer mode essentially influences the processes running in the weld pool that, in its turn, determines weld formation. Ensuring directed metal transfer in welding in different positions is also important. Therefore, this work sets forth the known theoretical approaches and describes the available mathematical models, allowing prediction of the main characteristics of metal transfer at different technological parameters of consumable electrode welding.

Methods of mathematical modelling of drop formation and electrode metal transfer in consumable electrode welding can be conditionally divided into two main groups (Figure 1). The first includes approaches, which enable prediction of just the individual characteristics of metal transfer process, such as drop size and detachment frequency. The main disadvantage of these models consists in that they do not allow determination of drop shape, or describing the phenomena of charge and energy transfer in molten electrode metal, which accompany the considered techno-

(C) A.P. SEMYONOV, 2014 logical process. The first group includes such procedures as static force balance theory (SFBT) [1-3], pinch instability theory (PIT) [4-6], as well as dynamic force balance theory (DFBT) $[7,8]$. The second group includes the model of drop formation in terms of hydrostatic approximation [9-11], as well as models based on equations of motion of viscous incompressible liquid. In its turn, in the subgroup of dynamic models thin jet approximation can be singled out [1214], as well as models based on total system of Navier-Stokes equations [15-20]. Let us consider the most widely accepted of the above methods.

SFBT. This method is based on analysis of the magnitude of resultant of forces, applied to molten metal drop. Drop dimensions and their detachment frequency are calculated, proceeding from detachment criterion. We assume that the drop has an axisymmetric shape and its detachment occurs at the moment, when the force, directed at drop detachment, is comparable by absolute value with the restraining force magnitude. Drop formation and detachment are determined by simultaneous action of forces of surface tension, gravity, electromagnetic, as well as aerodynamic force, due to mechanical interaction of metal drop with shielding gas flow. Surface tension force is calculated, using the following formula:

$$
F_{\mathrm{s}}=2 \pi R_{\mathrm{w}} \gamma,
$$

where $R_{\mathrm{w}}$ is the welding electrode radius; $\gamma$ is the coefficient of liquid metal surface tension. Note that during drop growth the axial compo- 


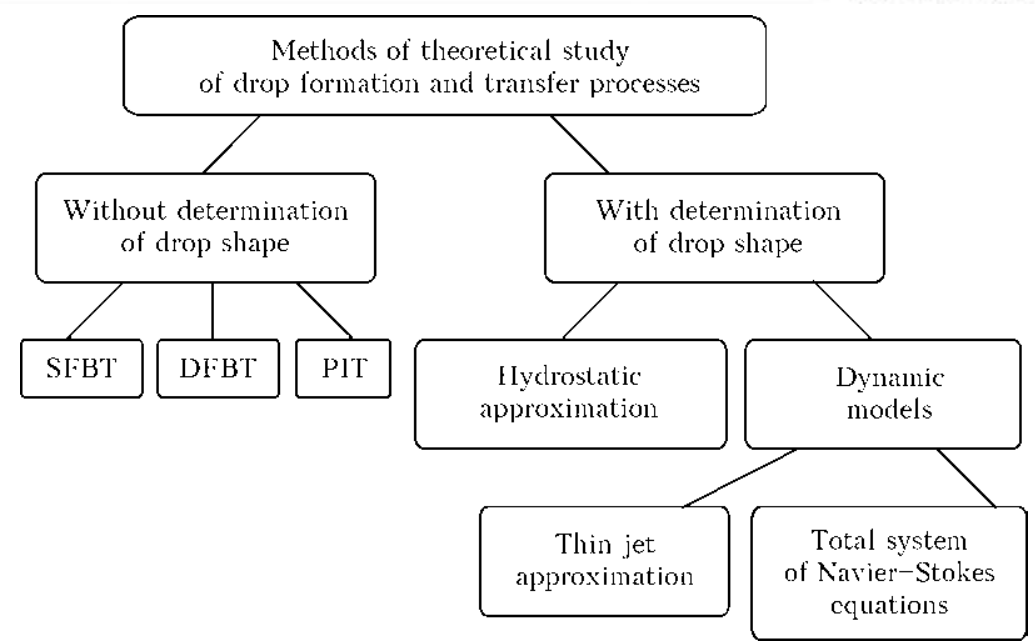

Figure 1. Classification of methods of theoretical study of the processes of electrode metal drop formation and transfer

nent of restraining force varies by magnitude, and formula (1) determines only its possible maximum value (upper estimate). Gravity force is calculated from the assumption that the drop has the shape of a sphere of radius $R_{\mathrm{d}}$ :

$$
F_{\mathrm{g}}=\frac{4}{3} \pi R_{\mathrm{d}}^{3} \rho g,
$$

where $\rho$ is the drop metal density; $g$ is the acceleration of gravity. Integral electromagnetic force is usually determined with application of an approach proposed in [21]. Let us write down the final expression for calculation of Lorenz force, acting on the drop:

$$
F_{\mathrm{em}}=\frac{\mu_{0} I^{2}}{4 \pi} \times
$$

$\times\left(\frac{1}{4}+\frac{1}{1-\cos \phi}-\frac{2}{(1-\cos \phi)^{2}} \ln \left(\frac{2}{1+\cos \phi}-\ln \left(\frac{R_{\mathrm{d}} \sin \phi}{R_{\mathrm{W}}}\right)\right)\right.$,

where $\mu_{0}$ is the magnetic constant, and angle $\phi$ determines the current-conducting region on the

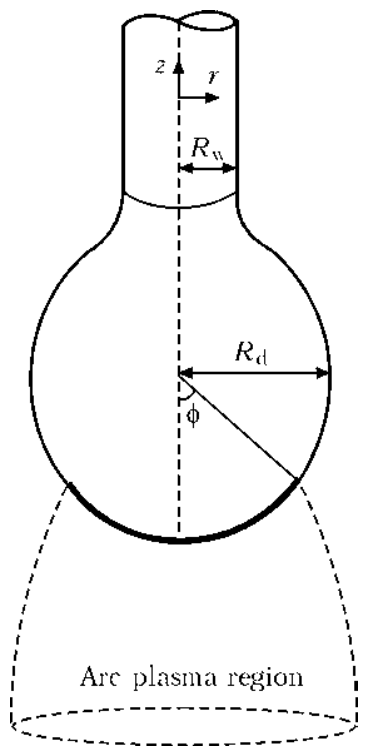

Figure 2. Schematic for analysis of SFBT method drop surface (region of arc anode binding) ( $\mathrm{Fi}^{-}$ gure 2).

Aerodynamic resistance is approximately calculated as a force applied to a sphere in gas flow:

$$
F_{\mathrm{a}}=\pi R_{\mathrm{d}} C_{D}-\frac{\rho_{\mathrm{g}} v_{\mathrm{g}}^{2}}{2},
$$

where $C_{D}$ is the coefficient of aerodynamic resistance; $\rho_{\mathrm{g}}, v_{\mathrm{g}}$ is the density and velocity of shielding gas flow, respectively. Dimensions of detached drop are found by solving non-linearity equation relative to unknown $R_{\mathrm{d}}$ value:

$$
F_{\mathrm{S}}=F_{\mathrm{a}}\left(R_{\mathrm{d}}\right)+F_{\mathrm{em}}\left(R_{\mathrm{d}}\right)+F_{\mathrm{g}}\left(R_{\mathrm{d}}\right) .
$$

Frequency of drop detachment is readily calculated at known values of wire feed rate and drop radius.

As can be seen, analysis of force factors influencing metal transfer characteristics is rather an estimate, as the drop surface is assumed to be spherical. Probably, this is exactly why the best agreement between experimental results and calculation data, derived by SFBT method, is observed in the case of globular metal transfer ( $\mathrm{Fi}^{-}$ gure 3 ). More over, when deriving expression

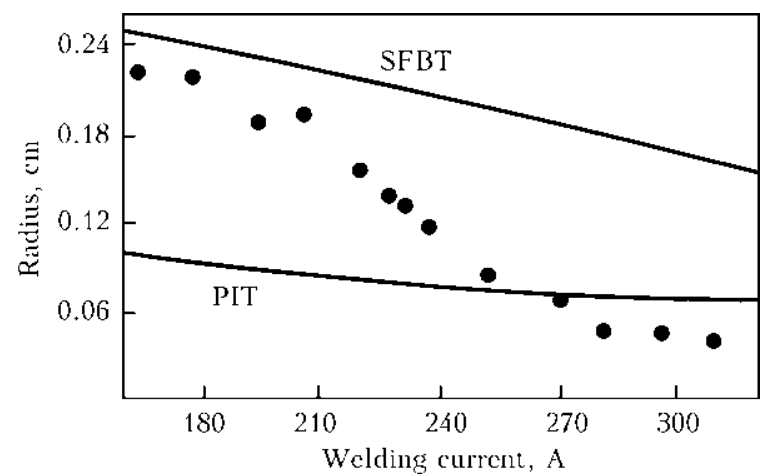

Figure 3. Comparison of experimental data (dots) with calculated ones (solid lines), obtained with SFBT and PIT methods [22], on dependence of dimensions of detached drops on current in welding with $1.2 \mathrm{~mm}$ steel wire in $\mathrm{Ar}+$ $+2 \% \mathrm{O}_{2}$ mixture 


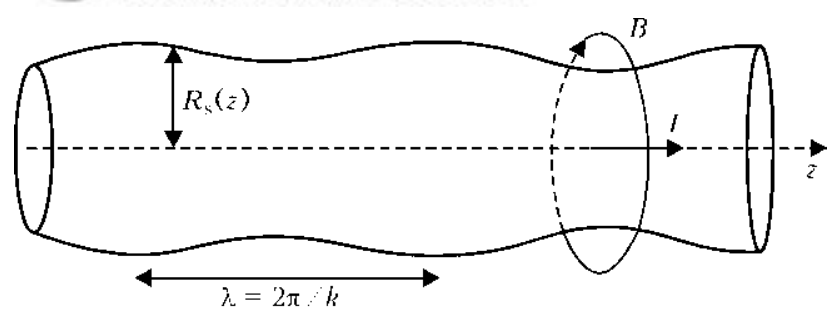

Figure 4. Instability of current-carrying liquid jet

(3), an assumption was made that current density is uniformly distributed in arc anode binding region. It should be also noted that within SFBT bounds, the influence of various kinds of shielding gas, or length of electrode extension on the process of drop formation and detachment cannot be substantiated.

DBFT. This model is similar to SFBT model in many respects. In this connection, this paper does not give its detailed description. Main difference of DFBT model from SFBT model consists in that it additionally allows for the forces of inertia.

PIT. Formation of electrode metal drops in terms of PIT model is treated as decomposition of a cylindrical liquid jet. This model is a generalization of Raleigh-Plateau theory of instability in the case of current-carrying jet. In keeping with works $[4,5]$ let us set forth the main postulates of this model. Let us consider an infinitely long liquid jet of radius $R_{0}$, through which electric current $I$ is running (Figure 4).

We will assume that the liquid surface undergoes a harmonic disturbance of small amplitude $\alpha$

$$
R_{\mathrm{S}}=R_{0}+\alpha e^{\omega t+i k z}, \quad \alpha<<R_{0},
$$

where $\omega$ is the parameter characterizing the velocity of disturbance increment; $k$ is the wave number of the considered mode. The following dispersion equation is derived by solving the linealized problem of magnetic hydrodynamics for a liquid jet:

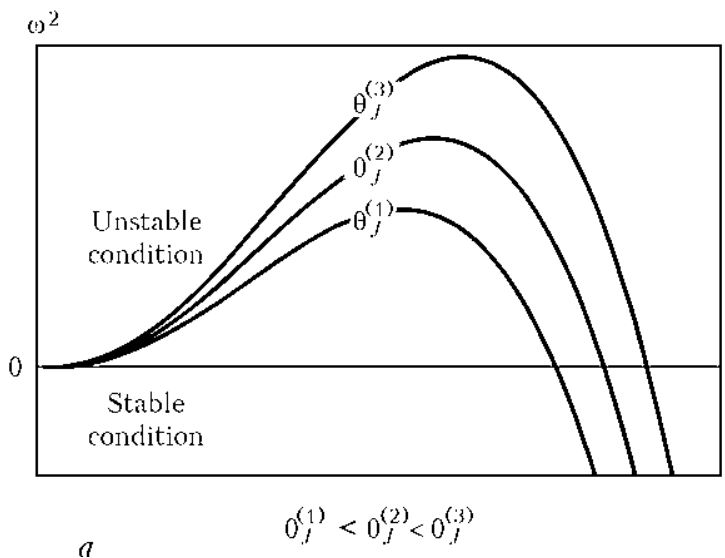

$$
\begin{gathered}
\omega^{2}=x\left(1-x^{2}\right) \frac{I_{1}(x)}{I_{0}(x)} \theta_{\gamma}+ \\
+\left(1+\frac{x}{2}\left(\frac{I_{1}(x)}{I_{0}(x)}-\frac{I_{0}(x)}{I_{1}(x)}\right)\right) \theta_{J}, \quad x=R_{0} k,
\end{gathered}
$$

where $\theta_{\gamma}=\gamma / \rho_{0}^{3} ; \quad \theta_{J}=\mu_{0} I^{2} / \rho \pi^{2} R_{0}^{4} ; \quad I_{n}(x), \quad n=$ $=0.1$ is the modified Bessel's function. One can see from Figure 5 that the disturbances, the length of which is smaller than $\lambda_{c}=x_{c} / 2 \pi R_{0}$ are decaying. As $\omega^{2}(x)$ dependence is nonmonotonic, there exist disturbance modes $\lambda_{m}$, to which maximum propagation rate corresponds.

Analysis of equation (7) allows determination of

$$
\left\{\begin{aligned}
\lambda_{c}= & \frac{2 \pi R_{0}}{0.923 \cdot \theta^{0.274}}, \\
\lambda_{m}= & \frac{2 \pi R_{0}}{0.625 \cdot \theta^{0.278}}, \\
\omega_{m}^{2}= & 0.616 \cdot \theta_{\gamma} \theta^{0.278},
\end{aligned}\right.
$$

where $\theta=\mu_{0} I^{2} / \pi^{2} \gamma R_{0}$. Further construction of PIT model is based on dependences (8). Let us assume that the length of jet section, from which the drop forms later on, is equal to $3 \lambda / 4\left(\mathrm{Fi}^{-}\right.$ gure 6). At transition from drop mode of metal transfer to spray mode, the molten electrode tip takes a tapered form (taper formation), as a result of which the drop base radius turns out to be much smaller than that of the wire. In the model, this factor is allowed for by geometrical parameter $\delta$ determining $R_{0}=\delta R_{\mathrm{w}}$ interrelation. Selection of value $\delta$ depends on the considered transfer mode. To study formation of large drops, values $\delta>1$ are used, whereas for spray mode $\delta<1$.

Let us write the resultant expressions, yielded by PTI model for drop volume $V_{\mathrm{d}}$ and their detachment frequency $f_{\mathrm{d}}$ :

$$
\begin{gathered}
V_{\mathrm{d}}=\frac{\pi R_{\mathrm{w}}^{2}}{2} \delta^{2} \lambda_{m}, \\
f_{\mathrm{d}}=\frac{2 v_{\mathrm{w}}}{\delta^{2} \lambda_{m}},
\end{gathered}
$$

where $v_{\mathrm{w}}$ is the wire feed rate.

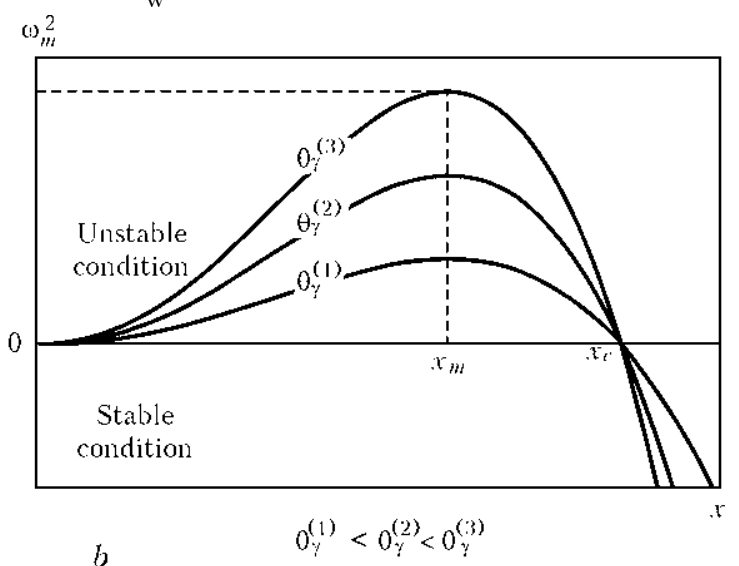

Figure 5. Influence of current $(a)$ and surface tension $(b)$ on velocity of instability propagation 
The best agreement between experimental data and results, derived with PIT method (see Figure 3 ), is observed in the case of large values of welding current $(I>240 \mathrm{~A})$, which are characterized by spray metal transfer. PIT model does not require any significant computational expenses or specialized software to determine the main characteristics of metal transfer. Similar to the previous model, however, PIT cannot clarify the influence of the kind of shielding gas or dimensions of electrode extension on the process of metal transfer. More over, at development of PIT method in works [4, 5] an important assumption was made that the total current is concentrated in the liquid jet volume, that is obviously not in agreement with experimental observations. Interrelation of value $\delta$ with other technological parameters of the welding process is also beyond the scope of the model. Work [6] is an attempt to correct some of the above disadvantages of the model: current flowing through the jet surface is taken into account, and jet effective radius $R_{0}$ is determined depending on current value.

Model of drop formation in terms of hydrostatic approximation. Numerous studies are devoted to the problem of finding the equilibrium form of liquid free surface in the gravity field and analysis of its stability, and their results are described in detail in [23, 24]. Analytical methods can be applied to solve this class of problems only in rare cases. As a rule, the body of numerical methods is used for this purpose (to find a solution). Theory of capillary surfaces can be readily generalized in the case of more complex processes, where electromagnetic forces are present and sometimes have the dominant effect, as well as forces generated by pressure of vapour, which evaporates from the free surface. Mathematical model of formation of electrode metal drop, allowing for the influence of electromagnetic forces, was proposed for the first time in [10]. Let us dwell on the main postulates of this model. The essence of the problem consists in determination of the shape and volume of an ultrastable drop, hanging from the electrode tip of specified radius. We will assume that the drop shape has the property of axial symmetry and can be described in the cylindrical system of coordinates as function $R=R(z), 0 \leq z \leq L_{\mathrm{d}}$, where $L_{\mathrm{d}}$ is the drop height. The considered model is based on hydrostatic equations

$$
\left\{\begin{array}{l}
\frac{\partial P}{\partial r}+j_{z} B=0 \\
-\frac{\partial P}{\partial r}+j_{r} B-\rho g=0
\end{array}\right.
$$

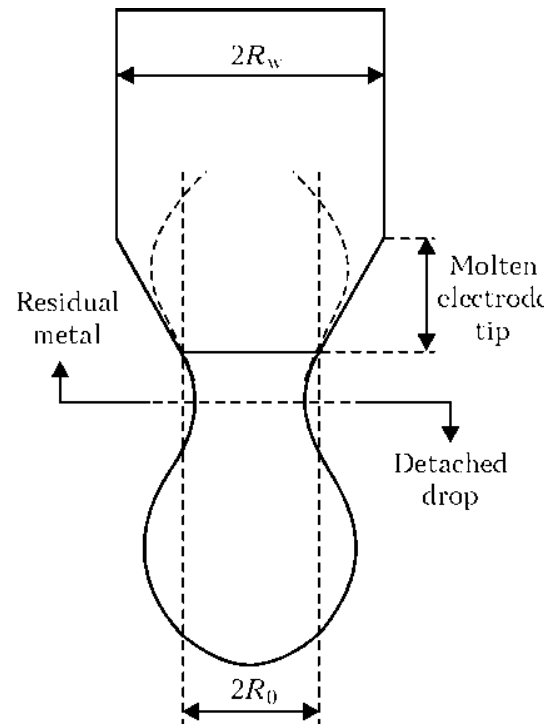

Figure 6. Schematic of drop formation in terms of PIT model [5]

and Laplace's law

$$
\gamma\left(\frac{1}{R_{1}}+\frac{1}{R_{2}}\right)=P(R, z),
$$

where $P$ is the pressure in liquid; $R_{1}, R_{2}$ are the main radii of surface curvature; $j_{r}, j_{z}$ are the components of current density vector; $B$ is the magnetic field induction. Main equations of the model have the following form:

$$
\left\{\begin{array}{l}
\frac{d P_{0}}{d z}=-\rho g+\frac{\mu_{0} R^{2}}{16} \frac{d}{d z}\left(\frac{I^{2}}{\pi^{2} R^{4}}\right), \\
P_{0}=\frac{\mu_{0} I^{2}}{4 \pi^{2} R^{2}}+\gamma \times \\
\times\left(-\frac{R^{\prime \prime}}{\left(1+R^{\prime 2}\right)^{3 / 2}}+\frac{1}{R\left(1+R^{\prime 2}\right)^{1 / 2}}\right), \\
0 \leq z \leq L_{\mathrm{d}},
\end{array}\right.
$$

where $P_{0}(z)=P_{0}(0, z)$ is the pressure on drop axis; $I=I(z)$ is the total current flowing in the drop section $z=$ const. Equations (13) are complemented by boundary conditions.

$$
\left.R\right|_{z=0}=0,\left.\quad R\right|_{z=L_{\mathrm{d}}}=R_{\mathrm{w}},
$$

as well as integral condition

$$
V_{\mathrm{d}}=\pi \int_{0}^{L_{\mathrm{d}}} R^{2} d z .
$$

When solving the problem, it is necessary to know how current, flowing through the drop cross-section, varies depending on height. In work [10] linear approximation $I(z)=I_{0} z / L_{\mathrm{d}}$ is used for this purpose. Size of integration range $L_{\mathrm{d}}$ is an unknown and should be determined. 


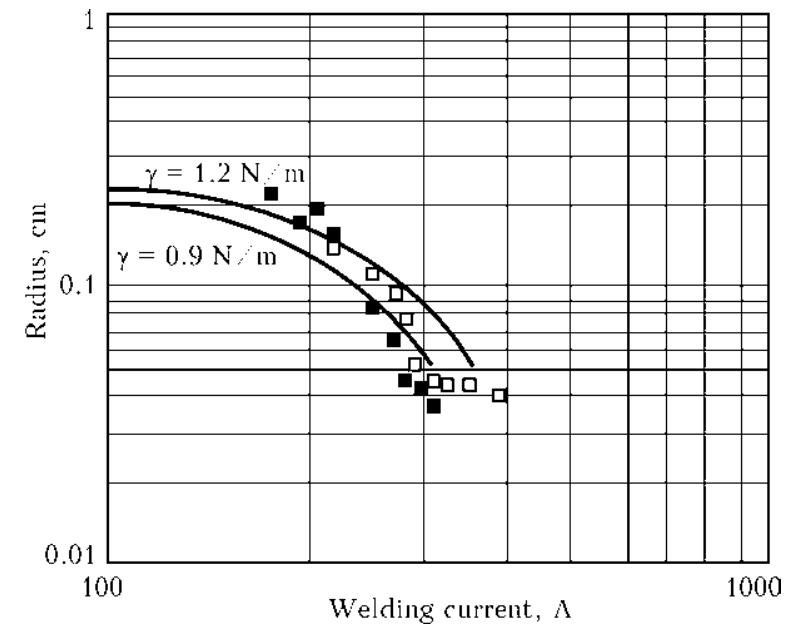

Figure 7. Dependence of detached drop radius on welding current: $\square, \square-$ experimental data of $[22,25]$, respectively; solid curves - numerical modelling [10]

Numerical solution of problem (13)-(15) is found using Runge-Kutta method. It is also important to note that such a solution is not unique for the specified wire radius and drop volume. From the multitude of solutions, it is necessary to select only the one which meets the criterion of stability [23]. In [10] comparison of experimental data $[22,25]$ for the detached drop radius with calculated values of similar quantity, generated using the considered model, is also performed (Figure 7). Numerical computations were conducted for welding low-carbon steel at $1.2 \mathrm{~mm}$ electrode diameter. As shown by computational experiments, for fixed electrode radius there exists threshold current value $I_{\text {th }}$, at which problem (13)-(15) does not have a stable solution. The author believes that this current value corresponds to transition of drop mode of metal transfer into the spray mode. An approximation dependence is proposed to calculate this parameter

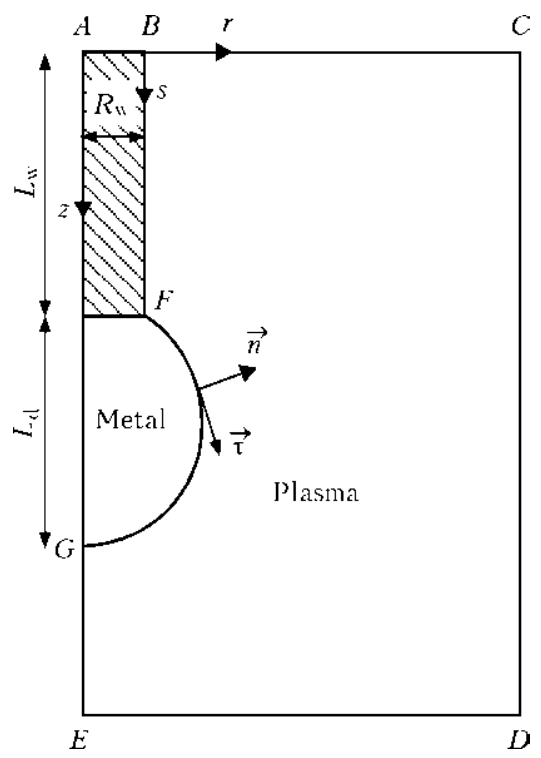

Figure 8. Schematic of calculation region

$$
I_{\mathrm{th}}=\sqrt{3.85 \cdot 4 \pi^{2} R_{\mathrm{w}} \gamma / \mu_{0}} .
$$

An important advantage of hydrostatic models is their relative simplicity. Performance of calculations on the base of these models does not require any significant computational resources. Such an approach, however, allows tracing drop formation just up to the stage of loss of stability. Processes of heat transfer in the metal volume also remain beyond the scope of the considered model. Thus, a more complete description of the process of drop formation at the tip of consumable electrode should be based on models describing hydrodynamic processes, proceeding in the drop.

Thin jet approximation. Model using total system of Navier-Stokes equations to describe hydrodynamic processes in the drop requires numerical realization of considerable computational resources that limits its application in a massive numerical experiment. Thin jet approximation [26], adapted to consumable electrode welding conditions [14], is considered as an alternative to this model. The following hypotheses were used in development of this model:

- melting front has a flat shape;

- wire feed rate corresponds to its melting rate;

- eddy motion of metal inside the drop is absent;

- arc column shape is assumed to be specified.

Model is based on the following system of equations:

$$
\left\{\begin{array}{l}
\frac{\partial V_{0}}{\partial t}+V \frac{\partial V_{0}}{\partial z}=-\frac{1}{\rho} \frac{\partial p_{0}}{\partial z}+\frac{3 v}{h^{2}} \frac{\partial}{\partial z}\left(h^{2} \frac{\partial V_{0}}{\partial z}\right)+g, \\
\frac{\partial h^{2}}{\partial t}+\frac{\partial\left(V_{0} h^{2}\right)}{\partial z}=0, \\
p_{0}=2 \gamma K+\int_{0}^{\infty} j_{z} B d r,
\end{array}\right.
$$

where $V_{0}=V_{0}(z, t)$ is the axial component of velocity on drop axis; $p_{0}=p_{0}(z, t)$ is the pressure on drop axis; $h=h(z, t)$ is the function describing the free surface shape; $K$ is the mean surface curvature. Initial and boundary conditions for equations (17) have the following form:

$$
\begin{gathered}
h(z, 0)=h_{0}(z), V_{0}(z, 0)=0, z \in\left(L_{\mathrm{w}}, L_{\mathrm{w}}+L_{\mathrm{d}}^{(0)}\right), \\
V_{0}\left(L_{\mathrm{w}}+L_{\mathrm{d}}, t\right)=\frac{d L_{\mathrm{d}}}{d t}, \quad V_{0}\left(L_{\mathrm{w}}, t\right)=v_{\mathrm{w}}, \\
h\left(L_{\mathrm{w}}+L_{\mathrm{d}}, t\right)=0, \quad h\left(L_{\mathrm{w}}, t\right)=R_{\mathrm{w}},
\end{gathered}
$$

where $L_{\mathrm{d}}^{(0)}, h_{0}(z)$ is the drop length and its surface shape in the initial condition, respectively. Solution of problem (17)-(19) is in the region of $L_{\mathrm{w}}+L_{\mathrm{d}} \geq z \geq L_{\mathrm{w}}$ (Figure 8).

Drop length, similar to the case of hydrostatic model, is to be determined. Calculation results, 

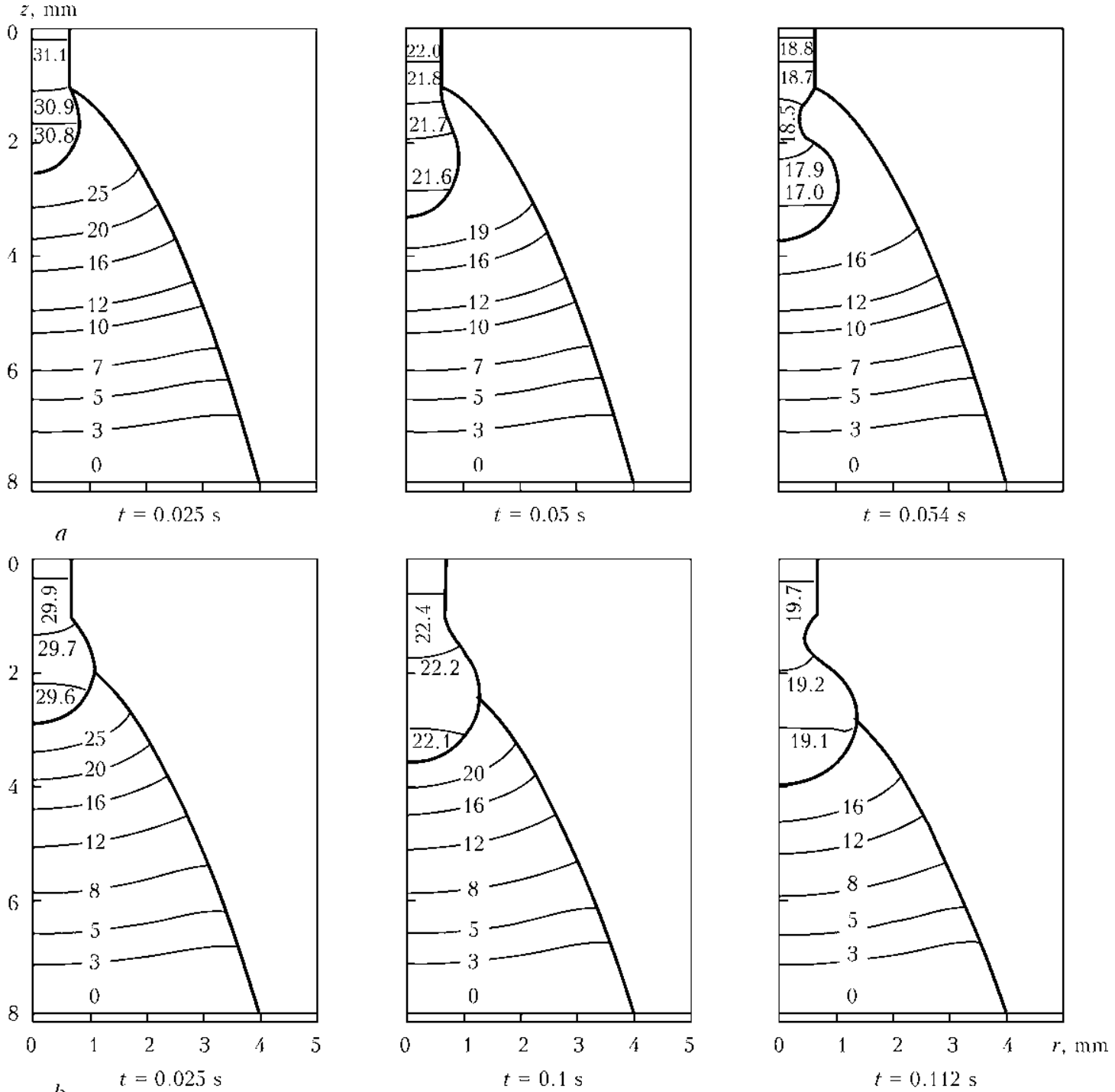

Figure 9. Dynamics of drop formation [14] for welding low-carbon steel in argon at $I=200 \mathrm{~A}, R_{\mathrm{W}}=0.6 \mathrm{~mm}$ and $v_{\mathrm{W}}=$ $4 \mathrm{~m} / \mathrm{min}$ : $a$ - region of arc binding corresponding to fusion boundary; $b$ - same, corresponding to maximum drop radius

obtained with the described model for two variants of arc binding to drop surface, are given in Figure 9.

Model of drop formation, based on thin jet equations, is a quite effective tool to obtain information about metal transfer characteristics. It, however, still does not allow describing a number of phenomena, having a dominant influence on heat transfer process. As shown by numerical estimates [27], metal inside the drop is involved into eddy motion, caused by Marangoni effect and influence of electromagnetic forces. Intensive stirring of drop metal is also confirmed by experimental studies [28]. As a result, convective mechanism of heat transfer in drop metal prevails over the heat conductivity process. As thin jet model does not allow description of effects associated with eddy motion of liquid metal, it does not seem possible to construct an adequate self-consistent model of drop formation on its basis.

Model of drop formation on the basis of total system of Navier-Stokes equations. Among the multitude of drop formation models, this model can be rightfully called the most complex one, as it coves the main physical factors influencing the process running. Moreover, development of computational algorithm to solve the respective equations involves a lot of difficulties. Let us write the mathematical formulation of the problem in the cylindrical system of coordinates:

$$
\left\{\begin{array}{l}
\rho\left(\frac{\partial u}{\partial t}+u \frac{\partial u}{\partial r}+v \frac{\partial u}{\partial z}\right)=-\frac{\partial P}{\partial r}+\mu\left(\Delta u-\frac{u}{r^{2}}\right)-j_{z} B, \\
\rho\left(\frac{\partial v}{\partial t}+u \frac{\partial v}{\partial r}+v \frac{\partial v}{\partial z}\right)=-\frac{\partial P}{\partial z}+\mu \Delta v+g \rho+j_{r} B,(20) \\
\frac{1}{r} \frac{\partial}{\partial r}(r u)+\frac{\partial v}{\partial z}=0 .
\end{array}\right.
$$


Here, $u, v$ are the radial and axial components of velocity vector $\vec{V}$, respectively; $\mu$ is the dynamic viscosity of drop metal. Integration domain $A B F G$ for equations (20) covers liquid metal zone and electrode solid part (see Figure 8). Let us assume that at initial moment of time $t=$ $=t_{0}$ there is no metal motion: $\left.\vec{V}\right|_{t=t_{0}}=0$. Boundary conditions for equations (20) have the following form:

$$
\begin{gathered}
\left.u\right|_{A G}=0,\left.\quad v\right|_{A B}=v_{\mathrm{w}}, \\
\left.\left(P-2 \mu \frac{\partial V_{n}}{\partial n}\right)\right|_{B F G}=2 \sigma K+P_{\mathrm{ev}}, \\
\left.\mu\left(\frac{\partial V_{n}}{\partial s}+\frac{\partial V_{\tau}}{\partial n}\right)\right|_{B F G}=\frac{\partial \gamma}{\partial s}, \quad\left(\left.\vec{V}\right|_{B F G}-\vec{V}_{\mathrm{s}}, \overrightarrow{n)}=0,\right.
\end{gathered}
$$

where $\vec{V}_{\mathrm{s}}$ is the surface motion velocity; $V_{n}, V_{\tau}$ is the projection of velocity vector on the normal and tangent to the surface, respectively; $P_{\mathrm{ev}}$ is the reactive pressure of metal vapours at convective evaporation. Note that conditions (22) express the balance of normal and tangential stresses on metal drop free surface. Kinematic condition (23) follows directly from mass balance on the surface. At numerical solution of hydrodynamics problem, solid phase viscosity is taken to be rather large $\left(10^{6}\right.$ times greater than that of liquid metal). This allows suppression of metal motion in the solid phase, and also eliminates the need for adapting the finite element net to melting front shape and setting additional boundary conditions on its surface. Such an approach was proposed for the first time in [29] and is found in literature under the name of «effective viscosity method». To solve problem (20)-(23), it is important to know electromagnetic field characteristics $j_{r}, j_{z}$, which, in their turn, are

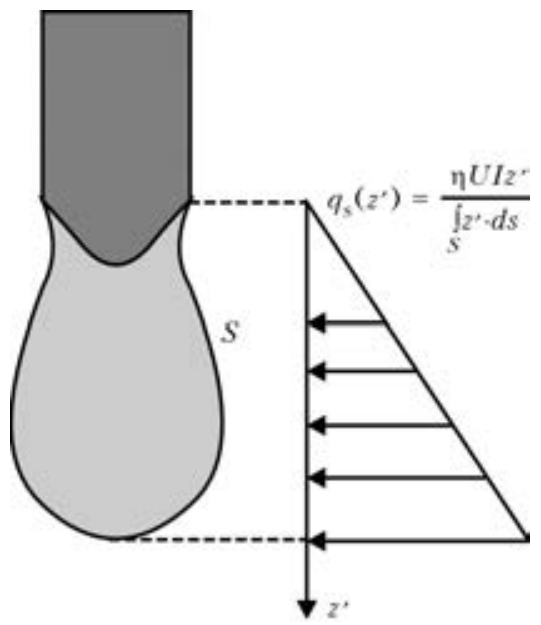

Figure 10. Heat flow distribution on the drop surface calculated through application of the model of electromagnetic processes:

$$
\begin{gathered}
(\nabla, \vec{j})=0, \\
\vec{j}=-\sigma \nabla \varphi, \\
{[\nabla, \vec{B}]=\mu_{0} \vec{j},}
\end{gathered}
$$

where $\sigma$ is the electric conductivity of the medium; $\varphi$ is the scalar potential of electric field. Solution of electrodynamics problem (24)-(26) is in $A C D E$ domain (see Figure 8) at superposition of the following boundary conditions:

$$
\left.j_{z}\right|_{A B}=I / \pi R_{\mathrm{w}}^{2},\left.\quad j_{z}\right|_{B C}=\left.j_{r}\right|_{C D}=0,\left.\quad \varphi\right|_{E D}=0 .
$$

Melt volume, penetration front shape and thermal condition of the liquid metal drop-wire solid section system are determined from solution of heat transfer equation

$$
\begin{gathered}
\frac{\partial H}{\partial t}+u \frac{\partial H}{\partial r}+v \frac{\partial H}{\partial z}= \\
=\frac{1}{r} \frac{\partial}{\partial r}\left(r \lambda \frac{\partial T}{\partial r}\right)+\frac{\partial}{\partial z}\left(\lambda \frac{\partial T}{\partial z}\right)+\frac{j^{2}}{\sigma},
\end{gathered}
$$

where $H$ is the specific enthalpy; $\lambda$ is the coefficient of heat conductivity. Solution (28) is in $A B F G$ domain at the following boundary and initial conditions:

$$
\begin{gathered}
\left.T\right|_{A B}=T_{0},\left.\quad \lambda \frac{\partial T}{\partial n}\right|_{B F G}=q_{\mathrm{s}}-q_{\mathrm{r}}-q_{\mathrm{c}}-q_{\mathrm{ev}} \\
\left.T\right|_{t=t_{0}}=T_{0}
\end{gathered}
$$

where $T_{0}$ is the initial wire temperature; $q_{\mathrm{s}}$ is the specific heat flow due to thermal impact of the $\operatorname{arc} ; q_{\mathrm{r}}, q_{\mathrm{ev}}, q_{\mathrm{c}}$ are the heat losses due to radiation, metal evaporation and convective heat exchange with shielding gas, respectively.

Volume of fluid (VOF) method [30] is the most wide-spread approach to solve the problem of drop formation (20)-(23). In VOF method the free surface is «spread» in the volume of a certain layer, thickness of which corresponds to several lengths of computational net cell, whereas surface tension force is considered as the volume force acting within this layer [31]. VOF algorithm is implemented in a number of applied software packages to solve hydrodynamics problems, such as ANSYS CFX, Fluent, Open Foam, etc. In addition, access to VOF initial program code is free [32] that allows researchers adapting it to solve problems related to metal transfer in welding. There exist also a number of other methods, where the free surface is considered as a boundary separated between two media. These methods form a group, which is found in litera- 
ture under the name of front traking methods (FTM) [33, 34]. Procedures with boundary separation allow a more detailed study of physical processes, occurring on the drop metal free surface. As an example, let us demonstrate application of FTM method to study drop formation and detachment in welding low-carbon steel in direct current mode. Physical properties of wire material and technological parameters, used in calculations, are given below.

Physical properties of material and process parameters Density $\rho, \mathrm{kg} \cdot \mathrm{m}^{-3}$ 7200

Viscosity $\mu, \mathrm{Pa} \cdot \mathrm{s} \ldots .006$

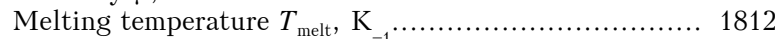

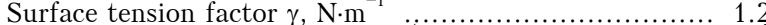

Electrical conductivity $\sigma, \mathrm{cm} \cdot \mathrm{m}^{-1} \ldots \ldots \ldots \ldots \ldots \ldots \ldots . .28 .54 \cdot 10^{3}$

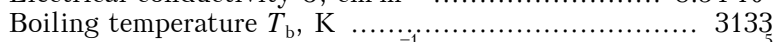

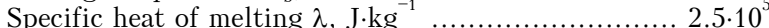

Specific heat capacity of the solid phase

$c_{\mathrm{sol}}, \mathrm{J} /(\mathrm{kg} \cdot \mathrm{K})$

700

Specific heat capacity of the liquid phase

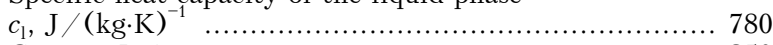

Current I, A ................................................. 350

Voltage $U, \mathrm{~V} \ldots \ldots \ldots \ldots . .50 .30 .5$

Wire feed rate $v_{\mathrm{w}}, \mathrm{m} \cdot \mathrm{min}^{-1} \ldots \ldots \ldots \ldots \ldots \ldots \ldots \ldots \ldots \ldots \ldots . \ldots . \ldots \ldots$

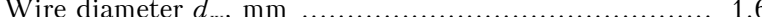

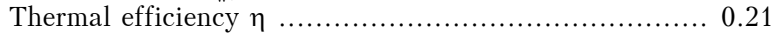

During numerical experiment it is assumed that heat flow $q_{\mathrm{s}}$ is distributed by a linear law (Figure 10). Calculated form of the drop, velocity field and melting front shape are shown in Figure 11.
As was already noted, formation of electrode metal drop is determined by interaction of a number of physical phenomena running in electrode metal, including a drop on its surface, as well as in arc plasma. Mathematical modelling methods enable studying the influence of each physical factor on the processes of drop formation and detachment separately. Mathematical models, considered in this work, allow determination of the main characteristics of electrode metal transfer (drop volume, detachment frequency), describing the dynamics of drop shape variation, as well as processes of heat and mass transfer in the melt. The most adequate description of the considered processes is provided by a model based on total system of Navier-Stokes equations. As shown by computational experiments, drop shape and its dimensions are largely determined by the action of electromagnetic force, the magnitude of which, in its turn, depends on the pattern of electric current flowing in the electrode metal drop-arc column system.

In conclusion, let us show the main directions of development of models of metal transfer processes, and also note the insufficiently studied aspects of physical phenomena, accompanying the process of consumable electrode welding. Studying the dynamics of neck thinning and breaking at drop detachment, in particular formation of
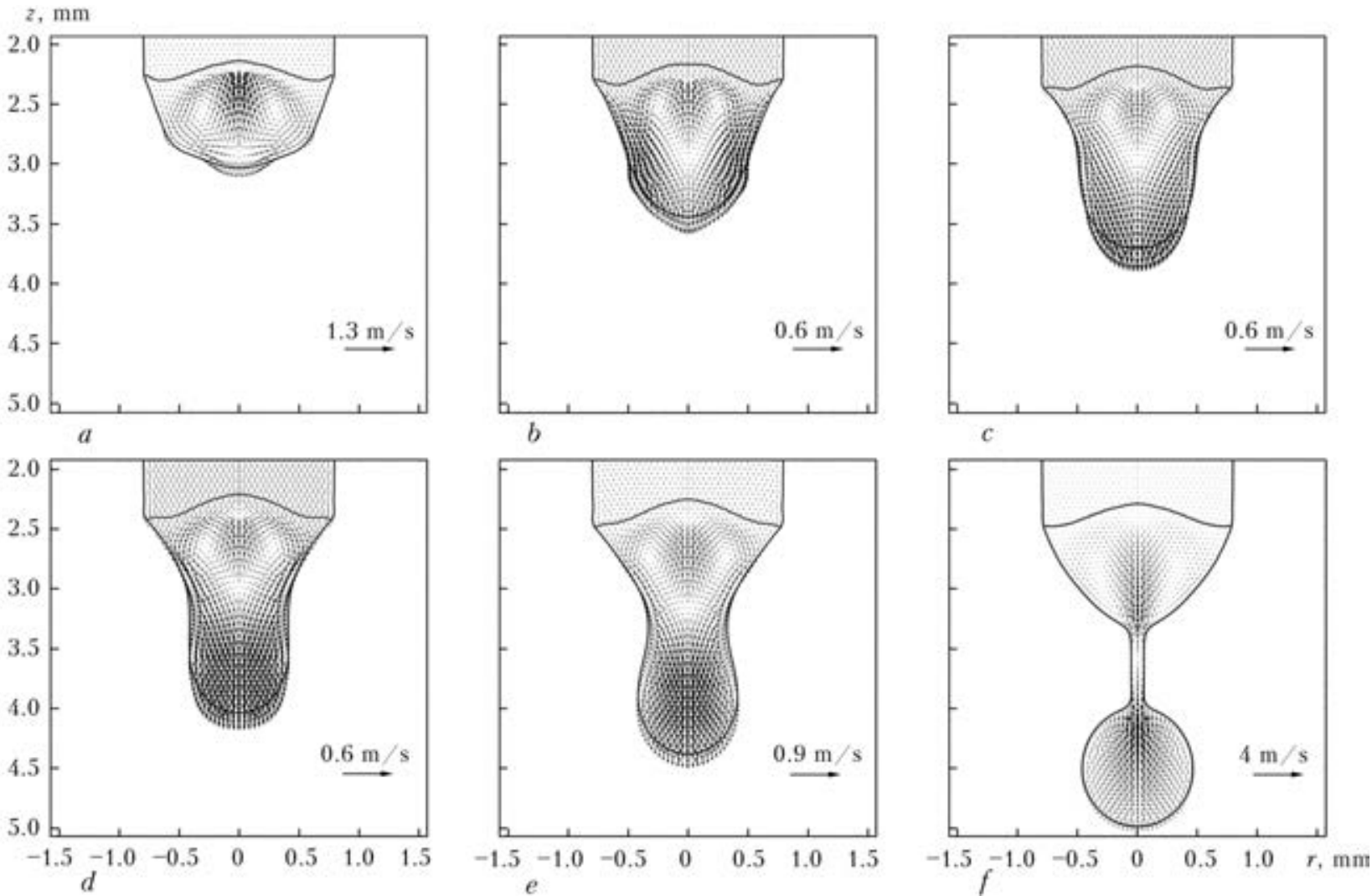

Figure 11. Drop shape and velocity field in the melt at different moments of time: $a-t=20.5 ; b-21.88 ; c-22.2$; $d-23.1 ; e-24 ; f-24.93 \mathrm{~ms}$ 
satellite drops, is of considerable interest, as well as physical processes running in the already detached drop [35]. In addition, hydrodynamic processes in the drop metal lead to emergence of convective diffusion of alloying elements [28]. Transport of alloying elements with a low boiling temperature, from fusion boundary to drop free surface, increases their evaporation intensity. This problem is not given sufficient attention in literature. Note that metal evaporation from the drop surface can not only affect the dynamics of its free surface, but also have a significant influence on the processes running in arc plasma. As shown by experimental studies [36], this factor is the cause for formation of a local minimum of plasma temperature in the central part of arc column. The above effect is also confirmed by theoretical studies [37]. Finally, in most of the studies, devoted to modelling electrode metal drop formation in consumable electrode welding, a constant value of surface tension factor is used, whereas it value changes significantly with temperature, and also depends on chemical composition of material and shielding gas. Modern methods of investigation allow determination of the coefficient of surface tension of liquid metals in a broad temperature range. At modelling of metal transfer, it allows studying the influence of Marangoni convection on the processes of heatand mass transfer in the drop.

1. Amson, J.C., Salter, G.R. (1962) An analysis of the gas-shielded consumable metal arc welding system. Brit. Welding J., 41(4), 232-249.

2. Greene, W.J. (1960) An analysis of transfer in gas shielded welding arcs. Pt 2. AIEE, 79(3), 194-203.

3. Waszink, J.H., Graat, L.H. (1983) Experimental investigation of the forces acting on a drop of weld metal. Welding J., 62(4), 109-116.

4. Allum, C.J. (1985) Metal transfer in arc welding as a varicose instability. Pt 1: Varicose instabilities in a current-carrying liquid cylinder with surface charge. J. Phys. D: Appl. Phys., 18(7), 1431-1446.

5. Allum, C.J. (1985) Metal transfer in arc welding as a varicose instability. Pt 2: Development of model for arc welding. Ibid., 18(7), 1447-1468.

6. Park, A.Y., Kim, S.R., Hammad, M.A. (2009) Modification of pinch instability theory for analysis of spray mode in GMAW. Ibid., 42(22), 225-503.

7. Choi, J.H., Lee, J., Yoo, C.D. (2001) Dynamic force balance model for metal transfer analysis in arc welding. Ibid., 34(17), 2658-2664.

8. Jones, L.A., Eagar, T.W., Lang, J.H. (1998) A dynamic model of drops detaching from a gas metal arc welding electrode. Ibid., 31(1), 107-123.

9. Voropaj, N.M., Kolesnichenko, A.F. (1979) Modeling of drop shape of electrode metal in gas-shielded welding. Avtomatich. Svarka, 9, 27-32.

10. Nemchinsky, V.A. (1994) Size and shape of the liquid droplet at the molten tip of an arc electrode. Phys. D: Appl. Phys., 27(7), 1433-1442.

11. Semyonov, A., Demchenko, V., Krivtsun, I. et al. (2010) Modelling of process of electrode metal droplet formation. In: Proc. of 5th Int. Conf. on Mathematical Modelling and Information Technologies in Welding and Related Processes (25-28 May 2010 Katsiveli, Crimea, Ukraine), 183-192. Kiev: PWI.

12. Semenov, O., Demchenko, V., Krivtsun, I. et al. (2012) A dynamic model of 'droplet formation in
GMA welding. Modelling and Simulation in Materials Sci. and Eng., 20(4), 045003.

13. Krivtsun, I.V., Semenov, O.P., Demchenko, V.F. (2011) To theory of electrode metal droplet formation in GMA welding. Dopovidi NANU, 6, 90-96.

14. Semenov, O., Demchenko, V., Krivtsun, I. et al. (2012) Modelling of the droplet formation process in GMA welding. In: Proc. of 10th Int. Sem. on Numerical Analysis of Weldability (Austria, Seggau, 2012), 83-94.

15. Haidar, J., Lowke, J.J. (1996) Predictions of metal droplet formation in arc welding. J. Phys. D: Appl. Phys., 29(12), 2951-2960.

16. Choi, S.K., Yoo, C.D., Kim, Y.S. (1998) Dynamic simulation of metal transfer in GMAW. Pt 1: Globular and spray transfer modes. Welding J., 77(1), 38-44.

17. Choi, S.K., Yoo, C.D., Kim, Y.S. (1998) Dynamic simulation of metal transfer in GMAW. Pt. 2: Shortcircuit transfer mode. Ibid., 77(1), 45-51.

18. Fan, H.G., Kovacevic, R.A. (2004) A unified model of transport phenomena in gas metal arc welding including electrode, arc plasma and molten pool. Ibid., 37, 2531-2544.

19. Wang, F., Hou, W.K., Hu, S.J. (2003) Modelling and analysis of metal transfer in gas metal arc welding. Ibid., 36, 1143-1152.

20. Hu, J., Tsai, H.L. (2007) Heat and mass transfer in gas metal arc welding. Pt 1: The metal. Int. J. Heat and Mass Transfer, 50, 808-820.

21. Amson, J.C. (1965) Lorentz force in the molten tip of an arc electrode. Brit. J. Appl. Phys., 16(8), 1169-1179.

22. Kim, Y.S., Eagar, T.W. (1993) Analysis of metal transfer in gas metal arc welding. Welding J., 72, 269-278.

23. Finn, R. (1989) Equilibrium capillary surfaces: Mathematical theory. Moscow: Mir.

24. Hartland, S., Hartley, R.W. (1976) Axisymmetric fluid-liquid interfaces: Tables giving the shape of sessile and pendant drops and external menisci, with examples of their use. Amsterdam: Elsevier Sci. Publ.

25. Rhee, S., Kannatey-Asibu, E. (1992) Observation of metal transfer during gas metal arc welding. Welding J., 71, 381-386.

26. Eggers, J., Dupont, T.F. (1994) Drop formation in a one-dimensional approximation of the Navier-Stokes equation. J. Fluid Mech., 262, 205-221.

27. Nemchinsky, V.A. (1997) Heat transfer in a liquid droplet hanging at the tip of an electrode during arc welding. J. Phys. D: Appl. Phys., 30(7), 1120-1124.

28. Wang J.B Nishimura, $H$, Katayama, $S$ et al. (2011) Evaporation phenomena of magnesium from droplet at welding wire tip in pulsed MIG arc welding of aluminium alloys. Sci. and Technol. of Welding and Joining, 16(5), 418-425.

29. Kou, S., Sun, D.K. (1985) Fluid flow and weld penetration in stationary arc welds. Metall. Trans act. $A, \mathbf{1 6}, 203-13$.

30. Hirt, C.W. Nichols, B.D. (1981) Volume of fluid (VOF) method for the dynamics of free boundaries. J. Comp. Phys., 39(1), 201-225.

31. Brackbill, J.U., Kothe, D.B., Zemach, C.A. (1992) A continuum method for modeling surface tension. Ibid., 100(2), 335-354.

32. Nichols, B.D., Hirt, C.W., Hotchkiss, R.S. (1980) SOLA-VOF. A solution algorithm for transient fluid flow with multiple free boundaries. NASA STI $/ R^{-}$ con Techn. Report, 81, 14281.

33. Wilkes, E.D. Philips, S.D., Basaran, O.A. (1999) Computational and experimental analysis of dynamics of drop formation. Phys. of Fluids, 11(12), 3577-3598.

34. Degroote, J., Bruggeman, P., Vierendeels, J. (2009) A coupling algorithm for partitioned solvers applied to bubble and droplet dynamics. Computers \& Fluids, 38(3), 613-624.

35. Nemchinsky, V.A. (2011) A droplet in the inter-electrode gap during gas metal arc welding. J. Phys. D: Appl. Phys., 44, 445203 .

36. Kozakov, R., Gott, G., Schopp, H. et al. (2013) Spatial structure of the arc in a pulsed GMAW process. Ibid., 46, 224001

37. Schnick, M., Hertel, M., Fuessel, U. et al. (2013) Energy balance in MIG arcs. Ibid., 46, 224002. 